\title{
Corrigendum: Targeting Anion Exchange of Osteoclast, a New Strategy for Preventing Wear Particles Induced-Osteolysis
}

\section{OPEN ACCESS}

Edited and reviewed by:

Maria Angela Sortino,

University of Catania, Italy

*Correspondence:

Degang YU

ydg163@126.com

Fengxiang Liu

liu_fengxiang@126.com

tThese authors have contributed equally to this work

Specialty section:

This article was submitted to Experimental Pharmacology and Drug Discovery

a section of the journal

Frontiers in Pharmacology

Received: 13 August 2019

Accepted: 23 August 2019 Published: 23 September 2019

Citation:

Wu C, Liu X, Sun R, Qin Y, Liu Z, Yang S, Tang T, Zhu Z, Yu D and Liu F (2019) Corrigendum: Targeting

Anion Exchange of Osteoclast, a

New Strategy for Preventing Wear

Particles Induced-Osteolysis.

Front. Pharmacol. 10:1078.

doi: 10.3389/fphar.2019.01078
Chuanlong Wu ${ }^{1,2 t}$, Xuqiang $\mathrm{Liu}^{3 t}$, Ruixin Sun ${ }^{4 t}$, Yunhao Qin ${ }^{5}$, Zhiqing Liu', Shengbing Yang ${ }^{1}$, Tingting Tang ${ }^{1}$, Zhenan Zhu ${ }^{1}$, Degang $\mathrm{Yu}^{1 *}$ and Fengxiang Liu ${ }^{1 *}$

${ }^{1}$ Shanghai Key Laboratory of Orthopaedic Implants, Department of Orthopaedics, Ninth People's Hospital, Shanghai Jiao Tong University School of Medicine, Shanghai, China, ${ }^{2}$ Department of Orthopaedics, Ruijin Hospital, Shanghai Jiao Tong University School of Medicine, Shanghai, China, ${ }^{3}$ Department of Orthopaedics, The First Affiliated Hospital, Nanchang University, Nanchang, China, ${ }^{4}$ State Key Laboratory of Oncogenes and Related Genes, Shanghai Cancer Institute, Renji Hospital, Shanghai Jiao Tong University School of Medicine, Shanghai, China, ${ }^{5}$ Department of Orthopaedics, Sixth People's Hospital, Shanghai Jiao Tong University, Shanghai, China

Keywords: bone resorption, wear particle, osteoclast, SLC4A2, actin

\section{A Corrigendum on}

Targeting Anion Exchange of Osteoclast, a New Strategy for Preventing Wear Particles Induced-Osteolysis

by Wu C, Liu X, Sun R, Qin Y, Liu Z, Yang S, Tang T, Zhu Z, Yu D and Liu F (2018). Front. Pharmacol. 9:1291. doi: 10.3389/fphar.2018.01291

In the original article, there was a mistake in Figure $\mathbf{1}$ as published. Figure $\mathbf{1 C}$ did not correctly match the description in the figure legend. The corrected Figure 1 appears below.

The authors apologize for this error and state that this does not change the scientific conclusions of the article in any way. The original article has been updated.

Copyright $\odot 2019 \mathrm{Wu}$, Liu, Sun, Qin, Liu, Yang, Tang, Zhu, Yu and Liu. This is an open-access article distributed under the terms of the Creative Commons Attribution License (CC BY). The use, distribution or reproduction in other forums is permitted, provided the original author(s) and the copyright owner(s) are credited and that the original publication in this journal is cited, in accordance with accepted academic practice. No use, distribution or reproduction is permitted which does not comply with these terms. 
A

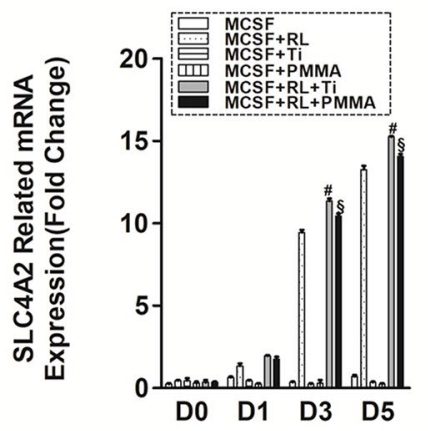

C

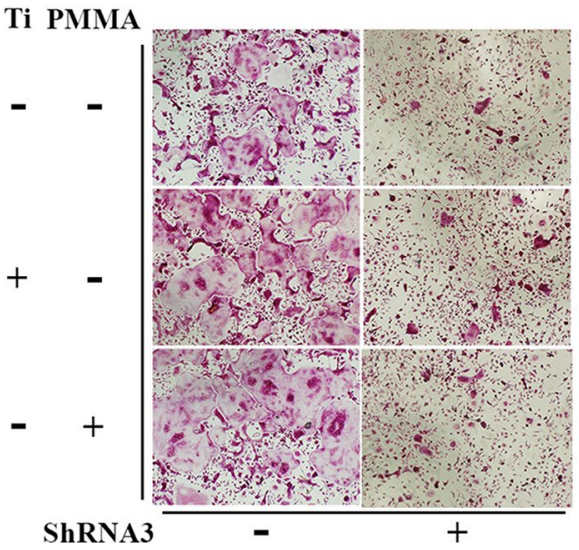

B

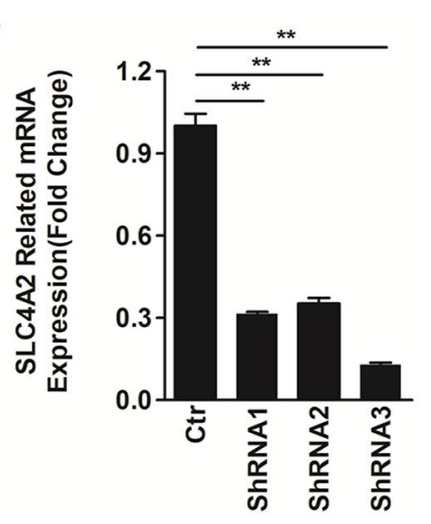

D

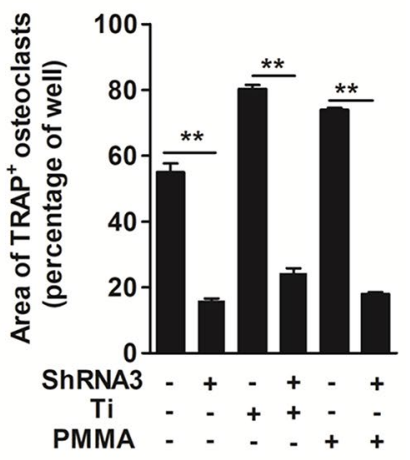

FIGURE 1 | SLC4A2 plays an important role in wear particle-induced osteoclastogenesis. (A) Up-regulation of S/c4a2 gene expression in the process of wear particle-induced osteoclastogenesis. Bone marrow-derived macrophages (BMM) were used $[\#, M C S F+R A N K L(R L)+T i$ vs. MCSF+RL, $P<0.01 ; \S$, MCSF+RL+PMMA vs. MCSF+RL, $P<0.01$ ]. (B) Three different shRNA vectors targeting S/c4a2, with shRNA3 yielding the greatest reduction in S/c4a2 mRNA. Effective knockdown of S/c4a2 in BMM cells, at $48 \mathrm{~h}$ after transfection using S/c4a2 shRNA1, shRNA2 and shRNA3, respectively. After transfection, cells were induced to differentiate into osteoclasts and harvested to examine S/c4a2 expression using reverse transcription quantitative PCR (RT-qPCR). (C) Effect of knockdown of S/c4a2, using shRNA3, on wear particle-induced osteoclastogenesis in vitro. (D) The area of TRAP-positive cells, measured using ImageJ ( ${ }^{\star} P<0.05$; $\left.{ }^{\star \star} P<0.01\right)$. At least three independent replicated of each experiment were conducted separately. 